\title{
Junction Flow between Drop Shaft and Diversion Tunnel in Lyss, Switzerland
}

\author{
Michael Pfister ${ }^{1}$ and Esther Rühli ${ }^{2}$
}

\begin{abstract}
A diversion tunnel is planned for flood protection in the City of Lyss, Switzerland. This tunnel includes a junction, from which water from a side creek is added to the tunnel flow. The latter is sensitive to choking, so that the junction had to be optimized in a hydraulic laboratory investigation. Its setup finally consists of a drop shaft and an injector generating annular flow in the tunnel. Free surface tunnel flow then occurred for all relevant discharges. This setup is also of interest in sewer systems as well as in spillway and diversion tunnels, where similar problems may occur. The concept was optimized for a specific prototype. General recommendations may be derived for similar situations. DOI: 10.1061/(ASCE)HY.1943-7900.0000365. @ 2011 American Society of Civil Engineers.
\end{abstract}

CE Database subject headings: Floods; Diversion structures; Shafts; Tunnels; Switzerland.

Author keywords: Diversion; Drop; Junction; Shaft; Tunnel.

\section{Introduction}

The Lyssbach River in northwestern Switzerland flooded the city of Lyss several times in recent years, causing damages of approximately 70 million Euro so far. In summer 2007, three major floods occurred. These were mainly caused by large discharges in combination with an insufficient discharge capacity of the river attributable to inadequate flow cross sections constrained by buildings and bridges. To improve this situation, a straight diversion tunnel was planned (Fig. 1). An intake structure upstream of the city partially collects the approach flow and discharges it into this tunnel. As a consequence, Lyssbach River only drains a reduced discharge to avoid damage within the city.

The diversion tunnel (subscript $T$ ) is $2.5-\mathrm{km}$ long, has a circular profile of diameter $D_{T}=4.28 \mathrm{~m}$ with a small bottom bench [Fig. 2(a)], and includes a smooth concrete lining of equivalent sand roughness height of $k_{s} \approx 1.2 \mathrm{~mm}$. The tunnel has a constant slope of $0.56 \%$. Its design discharge was based on the 100-years flood of $Q_{T, 100}=42.6 \mathrm{~m}^{3} / \mathrm{s}$, with a tunnel capacity discharge of $Q_{T, \mathrm{EF}}=$ $63.9 \mathrm{~m}^{3} / \mathrm{s}$, corresponding to $150 \%$ of the 100 -year flood, and subsequently denoted as extreme flood $(\mathrm{EF})$. The dimensionless tunnel discharge is expressed as $\Theta=Q /\left(g D^{5}\right)^{0.5}$ and is $\Theta_{T, 100}=0.36$ for the design discharge and $\Theta_{T, \mathrm{EF}}=0.54$ for EF. For all discharges, the tunnel has to operate under free surface conditions. Especially for large discharges, the tunnel operates with slightly supercritical flow, while definite supercritical flow occurs for smaller discharges. As shown in Fig. 2(b), the minimum Froude number is $F \cong 1.2$ for EF under uniform flow, with a filling ratio of $Y=h / D=0.69$,

${ }^{1}$ Lecturer, Laboratory of Hydraulic Constructions (LCH), Ecole Polytechnique Fédérale de Lausanne (EPFL), CH-1015 Lausanne, Switzerland; formerly, VAW, ETH Zurich (corresponding author). E-mail: michael .pfister@epfl.ch

${ }^{2}$ Research Engineer, Laboratory of Hydraulics, Hydrology and Glaciology (VAW), ETH Zurich, CH-8092 Zurich, Switzerland. E-mail: ruehli@vaw.baug.ethz.ch

Note. This manuscript was submitted on May 17, 2010; approved on November 15, 2010; published online on July 15, 2011. Discussion period open until January 1, 2012; separate discussions must be submitted for individual papers. This paper is part of the Journal of Hydraulic Engineering, Vol. 137, No. 8, August 1, 2011. CASCE, ISSN 0733-9429/2011/8$836-842 / \$ 25.00$. i.e., close to the maximum under these conditions according to Sauerbrey (1969) or Gargano and Hager (2002). The approximation $\mathrm{F}=Q /\left(g D h^{4}\right)^{0.5}$ (Hager 1999) applies exclusively for the circular profile, whereas the present tunnel has a small bench at its bottom. The latter provides an access road for maintenance [Fig. 2(a)]. Its effect was accounted for by using the exact relation $\mathrm{F}^{2}=\left(Q^{2} / g A^{3}\right)(d A / d h)$ (Chow 1959), where $Q=$ discharge; $g=$ gravitational acceleration; $A=$ flow cross section [shaded area in Fig. 2(a)]; and $h=$ flow depth. The free surface width corresponds to $b=(d A / d h)$, so that $\mathrm{F}=V /(g A / b)^{0.5}$, with $V=$ flow velocity.

At $1.6 \mathrm{~km}$ downstream of the tunnel inlet, Grentschelbach (subscript $G$ ) Creek crosses the tunnel axis about $10 \mathrm{~m}$ above the tunnel ceiling (Fig. 1). This creek also has to be drained, because it flooded portions of the city during intense rainfalls. During floods, a portion of its discharge should be directed into the tunnel via a side channel, connected to a 1.25 -m-diameter conduit of constant slope of $1.5 \%$. A 100-year flood corresponds to $Q_{G, 100}=3.4 \mathrm{~m}^{3} / \mathrm{s}$, whereas $Q_{G, \mathrm{EF}}=1.5 Q_{G, 100}=5.1 \mathrm{~m}^{3} / \mathrm{s}$. There, $\Theta_{G, 100}=0.03$ for design discharge and $\Theta_{G, \mathrm{EF}}=0.04$ for $\mathrm{EF}$, both defined with $D_{T}$ as the reference diameter. Uniform flow conditions of the conduit correspond to $Y=0.58$ and $\mathrm{F}=1.8$ for design discharge, whereas $Y=0.82$ and $\mathrm{F}=1.4$ for $\mathrm{EF}$. The conduit bottom joins the drop shaft to the diversion tunnel $7.45 \mathrm{~m}$ above the tunnel ceiling.

A junction combined with a vertical drop is a hydraulically sensitive structure, as (1) the discharge capacity of both branches has to be guaranteed, especially because of supercritical tunnel flow with high filling ratios; (2) undesirable flow phenomena such as tunnel choking, flow pulsations, or a choking of the creek inlet should be avoided; and (3) the tunnel diameter must remain constant within the junction for constructional ease. A first draft of the junction included a vortex drop shaft with a dissipation chamber, supplying the flow to the tunnel via a free overfall [Fig. 3(a)]. This setup generated poor flow features, however, such that an improved design had to be developed. This case study describes the related optimization process and its result: a drop shaft combined with an injector.

\section{Hydraulic Model}

The discharge characteristics of the drop shaft and the junction were tested and optimized in a hydraulic model at the Laboratory 


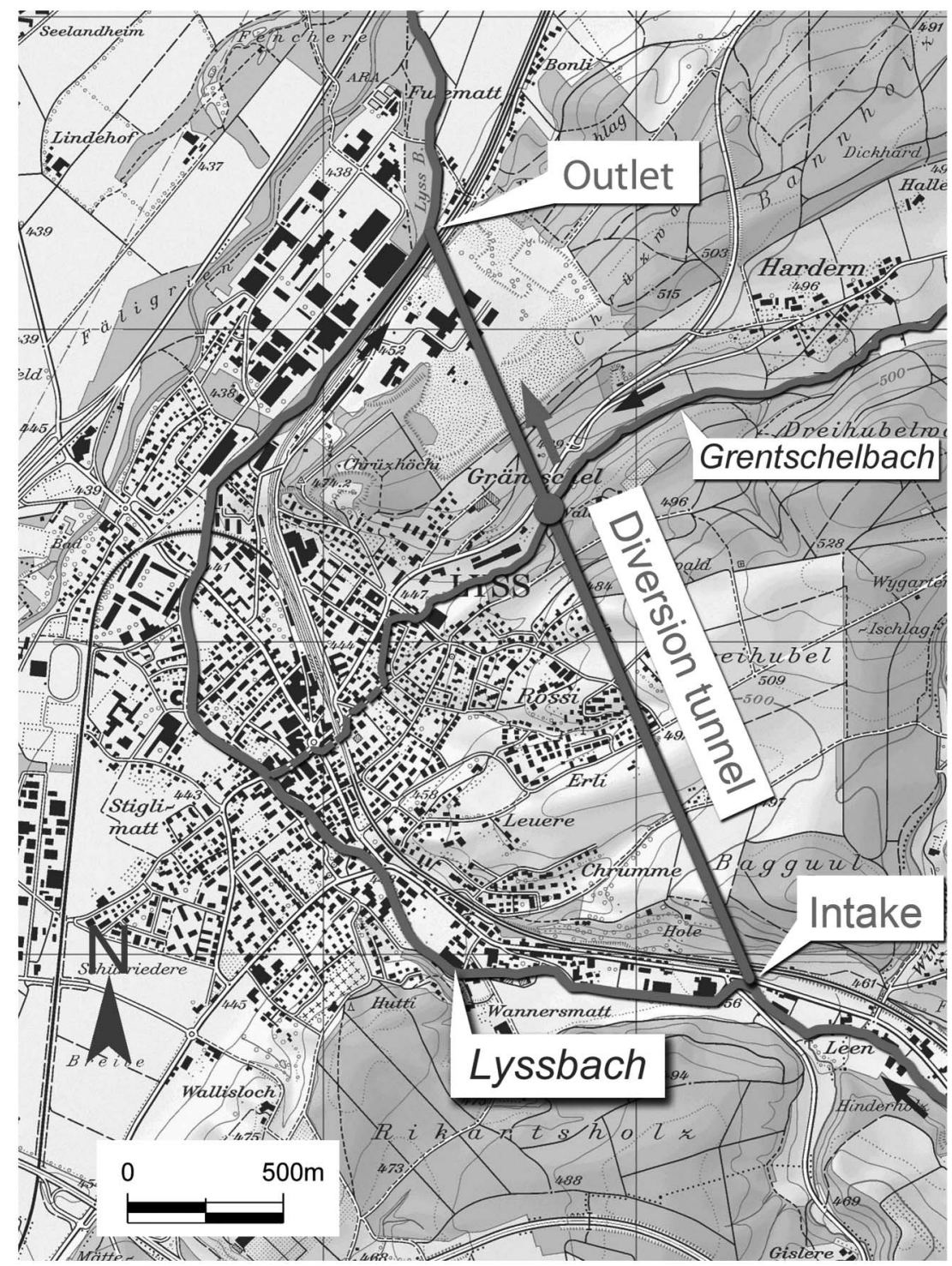

Fig. 1. Overall arrangement of flood protection works at city of Lyss [reproduced with permission from swisstopo (JA100120)]

of Hydraulics, Hydrology and Glaciology (VAW) of ETH Zurich. The model involved Froude similitude with a length scale of 1:17 (Laboratory of Hydraulics, Hydrology and Glaciology, Zurich, Switzerland, unpublished report, 2010) consisting of a 170-m long tunnel section, the creek conduit, the drop shaft, and the connection between the latter and the tunnel. All model elements were made of Perspex or PVC. The tunnel and the creek discharges were measured with an electromagnetic flowmeter with $\pm 0.5 \%$ accuracy. The pressure heads were measured to the next millimeter with taps, located at the tunnel bottom with a streamwise spacing of $1.0 D_{T}$ (at every symbol of Figs. 4 and 5), at the sidewalls, and on the bottom of the dissipation chamber or the injector. The measured bottom pressure heads are, in general, equal to the flow depths because the tunnel slope and the air entrainment at the junction are small involving nearly hydrostatic conditions, with $\mathrm{F}_{T}=1.4$ for design discharge under uniform flow. Flow features were observed visually and documented by photographs. Scale effects concerning flow losses in the tunnel are absent because the friction factors are equal in the model and the prototype, whereas the air entrainment in the tunnel and at the shaft is slightly underestimated. For plunging jets as occur in the final design, Chanson (2009) proposed a minimum Weber number $\mathrm{W}=\rho V^{2} d / \sigma$ of $10^{-3}$ to avoid significant scale effects related to air entrainment, with $\rho=$ fluid density; $\sigma=$ fluid surface tension; and $d=$ jet diameter. If considering a conservative jet diameter of $d=0.45 \mathrm{~m}$ equal to the injector height, as shown in Fig. 3(e), $\mathrm{W}=2.6 \times 10^{-3}$ results for design discharge and $\mathrm{W}=3.9 \times 10^{-3}$ for $\mathrm{EF}$ for the jet generated by the injector at the junction. The aforementioned limitations are accordingly just respected.

\section{Preliminary Options}

\section{Vortex Drop Shaft}

Option A consists of a vortex drop shaft with an inlet structure according to Hager (1990), including a vertical drop shaft (subscript $S$ ) of diameter $D_{S}=1.50 \mathrm{~m}$; a dissipation chamber of length $5.3 D_{S}$, width $1.4 D_{S}$ and height $2.9 D_{S}$; and a free overfall connecting the chamber to the tunnel (Volkart 1993) [Fig. 3(a)]. The chamber joins the tunnel with a horizontal angle of $45^{\circ}$. The connecting cross section between the chamber and the tunnel is rectangular of height $s=1.2 h_{c}$, with $h_{c}=\left(q_{G, \mathrm{EF}}^{2} / g\right)^{1 / 3}$ and $q=$ specific discharge. The overfall crest is located at $Y=0.70$ above the tunnel 


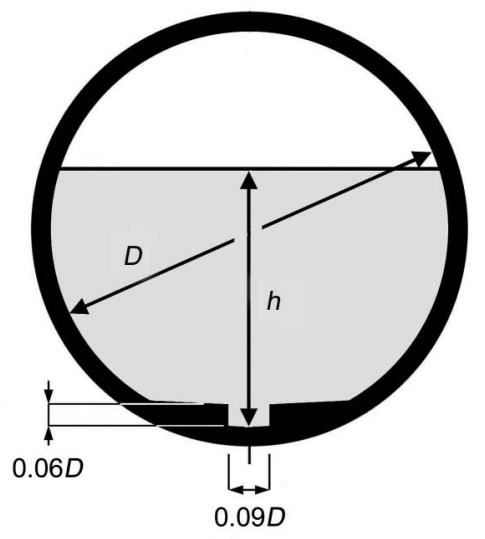

(a)

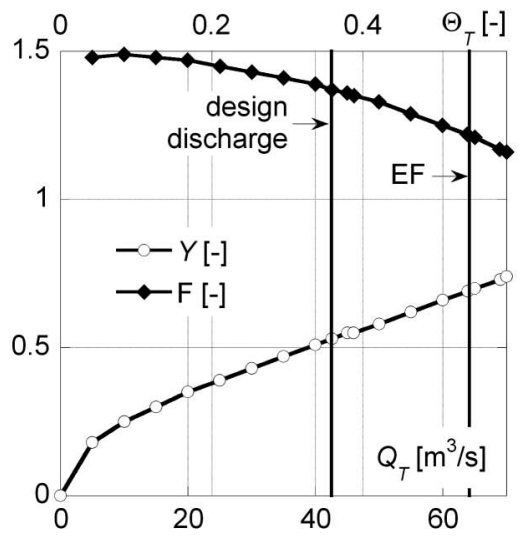

(b)

Fig. 2. (a) Tunnel cross section with bench and notation; (b) hydraulic characteristics of diversion tunnel flow

bottom, equivalent to a tunnel flow depth under $Q_{T \text {.EF }}$. Accordingly, the tunnel flow is undisturbed as long as there is no lateral inflow, i.e., $Q_{G}=0 \mathrm{~m}^{3} / \mathrm{s}$.

The pressure head of turbulent two-phase air-water flows measured in the dissipation chamber is $2.2 D_{S}$ under design discharges of both branches and $2.3 D_{S}$ for EF of both branches, resulting in filling ratios of $Y=0.76$ and 0.80 , respectively. Because of intense air entrainment, the two-phase flow surface touches the chamber ceiling if at least one branch has EF discharge. The chamber was submerged, even though the recommendations of Hager (1999) were respected, as a result of the narrow slot and the tunnel flow effect.

The tunnel flow was disturbed as soon as $Q_{G}>0 \mathrm{~m}^{3} / \mathrm{s}$. Local choking resulted under EF condition of both branches, whereas it was close to choking under the design discharge condition, as shown in Fig. 4. There, the streamwise tunnel coordinate is $x=0$ at the junction. The disturbed flow extends to the upstream model boundary under the EF condition, while the corresponding length is almost $10 D_{T}$ under the design discharge condition. A weak hydraulic jump occurred at the transition from the upstream supercritical tunnel flow to the disturbed reach. Downstream of the junction, the flow was again supercritical with a free surface and shock waves for the combined operation of both branches. In the vicinity of the junction, the effective water surface was slightly above that shown in Fig. 4 because the air entrained by the lateral inflow locally affects the tunnel bottom pressures. As the flows in both the tunnel and the dissipation chamber were unacceptable, the junction was modified.
Option $B$ is similar to option A, except for the junction slot located at the tunnel bottom [Fig. 3(b)]. The tunnel flow is then only insignificantly disturbed by the junction if $Q_{G}=0$. The dissipation chamber still chokes for the EF condition of both branches. The air transport from the dissipation chamber to the tunnel was reduced as compared with option A, as the moderately deaerated flow close to the chamber bottom migrates into the tunnel. The tunnel filling ratios are similar to these of option A (Fig. 4). Again, the tunnel flow chokes for the EF discharge condition of both branches.

A slot height of $s=0.4$ and $0.8 h_{c}$, next to $1.2 h_{c}$ previously considered, combined with an unchanged slot width, did not fundamentally affect the flow pattern. However, gated flow results in higher flow depths in the dissipation chamber, which is negative relating to choking. The tunnel flow depth increases slightly for smaller slot heights, so that the length of the disturbed reach increases as well.

Option $C$ is similar to options $\mathrm{A}$ and $\mathrm{B}$, but the slot elevation was fixed at mid tunnel height [Fig. 3(c)], so that no spiral component is superposed to the tunnel flow. The alignment of the combining flows was thereby slightly improved. Furthermore, the disturbance of the tunnel flow surface was reduced as compared with the upper or lower slot elevations, as previously described. However, the general flow features in the dissipation chamber and in the tunnel (Fig. 4) were almost identical to these of options A and B.

The vortex drop shaft concept was therefore rejected on the basis of these model tests. The velocity of the creek inflow was too small as compared with the tunnel flow conditions. Higher velocities require a higher flow energy, which is not available downstream of a dissipation chamber, if submergence or lifting of the bottom elevation are excluded. For the latter, the drop shaft height is less than $7 \mathrm{~m}$, which is considered as a minimum value (Hager 1999).

\section{Injectors}

Option $D$ includes several setups and investigates injectors at the tunnel ceiling with pressurized shaft flow [Fig. 3(d)]. To increase flow velocities, the injector cross sections were reduced as compared with previous test cases. Simultaneously, the horizontal supply angle was reduced to minimize the transverse flow component. To ascertain the required energy head for optimum injector flow conditions, the drop flow features were a priori not considered for the basic tests. An optimum of the injector shape, the tunnel flow features, and the required energy head in the drop resulted by respecting the available elevation difference between the tunnel ceiling and the conduit bottom.

A first test series consisting of circular injectors indicated that a cross section of $0.45 \mathrm{~m}^{2}$ represents both an optimum relating to the injector velocity and a minimum disturbance of the tunnel flow, with regard to the available energy head. To exploit the available energy head for large discharges, a smooth shaft geometry involving small flow losses is required. A second test series investigated injectors of rectangular cross sections. For wide injectors of similar cross-sectional areas as the circular devices, the height reduces, resulting in a thinner water jet on the tunnel surface. The disturbance of the tunnel flow is then significantly reduced. A third series included injectors merging the tunnel ceiling either tangentially or with a small offset. The offset was intended to allow for longitudinal tunnel air flow, connecting the regions up and downstream of the junction. The lower injector jet boundary, however, was located closer to the tunnel flow surface so that tunnel choking already occurred for small $Q_{T}$. In contrast, the jet attaches to the tunnel surface for setups without offset, resulting only in a minimum tunnel flow disturbance. Fig. 4 compares the free surface profiles 

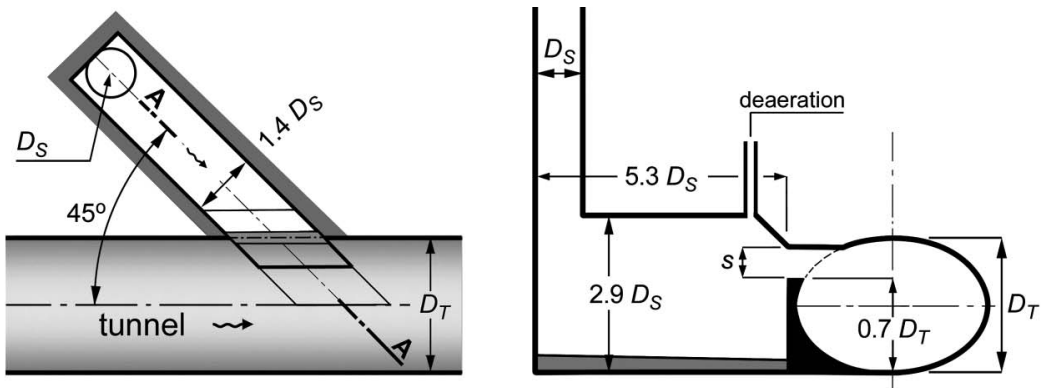

(a)
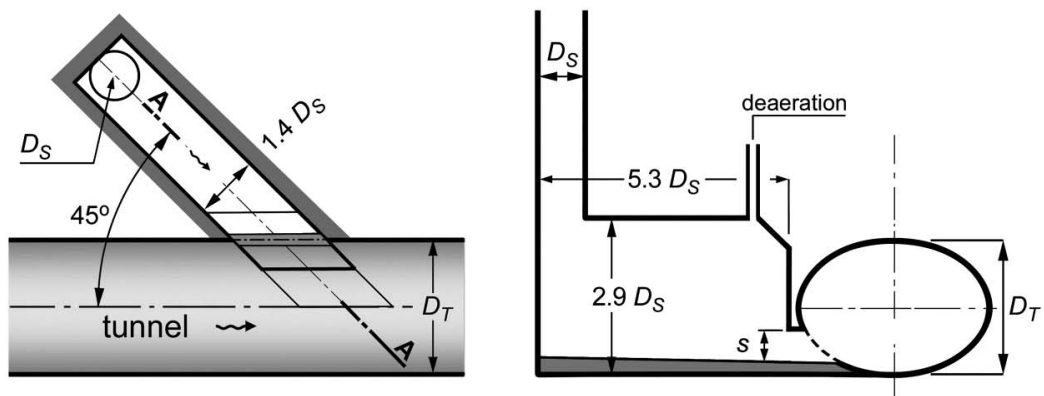

(b)
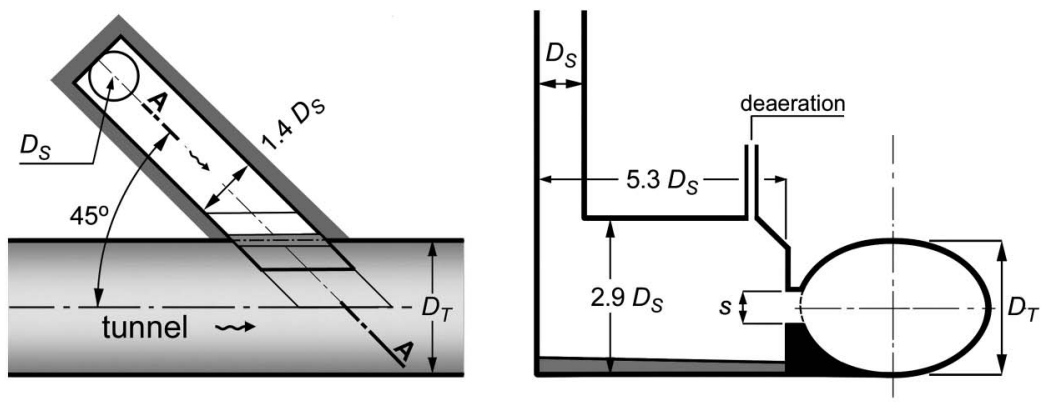

(c)
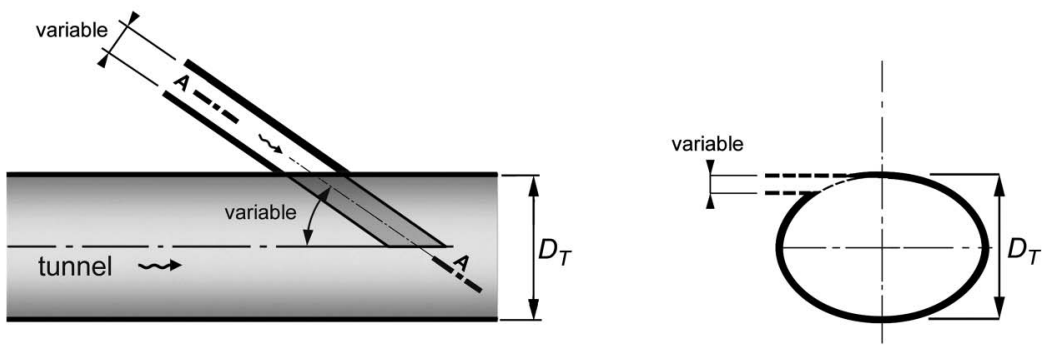

(d)

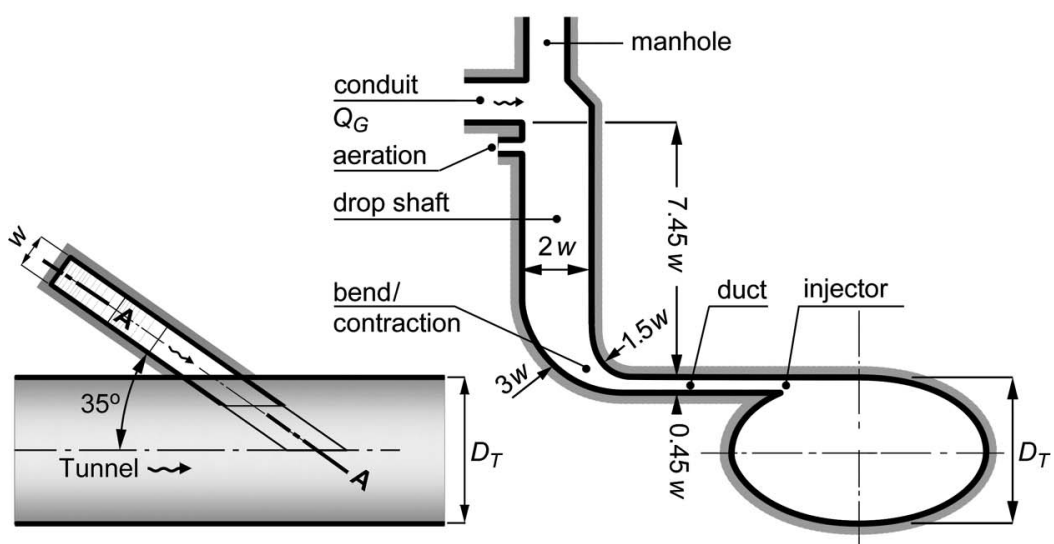

(e)

Fig. 3. Schematic design of options: (a) A; (b) B; (c) C; (d) D; (e) final design, left plan view and right section A-A 


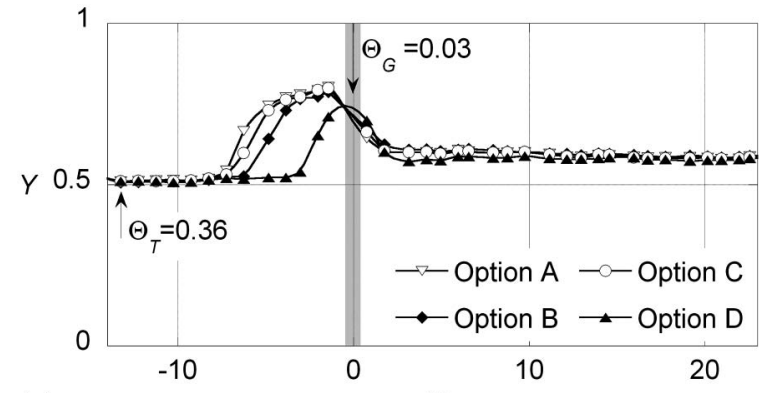

(a)

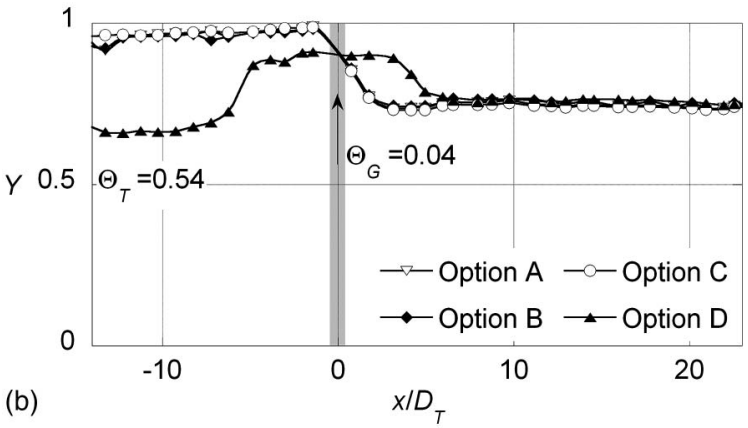

Fig. 4. Tunnel flow surface profiles $Y\left(x / D_{T}\right)$ at junction vicinity under options A to D: (a) design discharge condition; (b) EF condition

$Y(x / D)$ of option $D$ consisting of a rectangular injector of height $0.5 w$ and width $1 w$ located at the tunnel ceiling with previous options. The junction effect on the tunnel flow was significantly reduced, especially under the EF condition of both branches.

The experiments furthermore indicated that the combination of a vortex drop inlet structure (Hager 1999) generating annular flow in the vertical shaft with a bend at its end and an injector resulted in submerged shaft and choked tunnel flow, which is unacceptable. The residual flow energy at the injector was, accordingly, too small even if a dissipation chamber at the shaft toe was absent. The classical vortex drop inlet construction and the round shaft were hence replaced by a different concept.

\section{Drop Shaft Combined with Injector}

The option resulting from the optimization process [Fig. 3(e)] includes the conduit close to the surface, a circular free end-overfall into the vertical drop shaft, a bend combined with a contraction to a horizontal duct, and an injector at the tunnel ceiling. The vertical drop shaft of rectangular horizontal cross section is $w=1.0 \mathrm{~m}$ wide and $2 w$ long. The conduit diameter is $1.25 \mathrm{~m}$ and thus wider than the shaft, such that a smooth transverse contraction is provided in the upper shaft portion. The vertical shaft height, including the bend, is $7.45 w$. The bend has an inner radius of $1.5 \mathrm{w}$ and an outer radius of $3 w$, including a streamwise cross-sectional contraction. The horizontal duct has the same width $w$ as the shaft, but a reduced height of $0.45 w$ as compared with the shaft length of $2 w$. For geometrical reasons, its length is $7.2 w$. The upper injector boundary is located tangentially at the tunnel ceiling without offset.

This design minimizes the tunnel flow disturbance and particularly avoids flow choking, thus guaranteeing the required discharge capacity under the EF condition. The injector velocity $V_{G}$ is accelerated to roughly $7.6 \mathrm{~m} / \mathrm{s}$ for design discharge, corresponding to $V_{T}=V_{G} \cdot \cos \left(35^{\circ}\right)=6.2 \mathrm{~m} / \mathrm{s}$ along the tunnel axis, where $V_{T}=5.7 \mathrm{~m} / \mathrm{s}$ was measured for the design discharge condition. The injector width was given by the shaft width, whereas its height
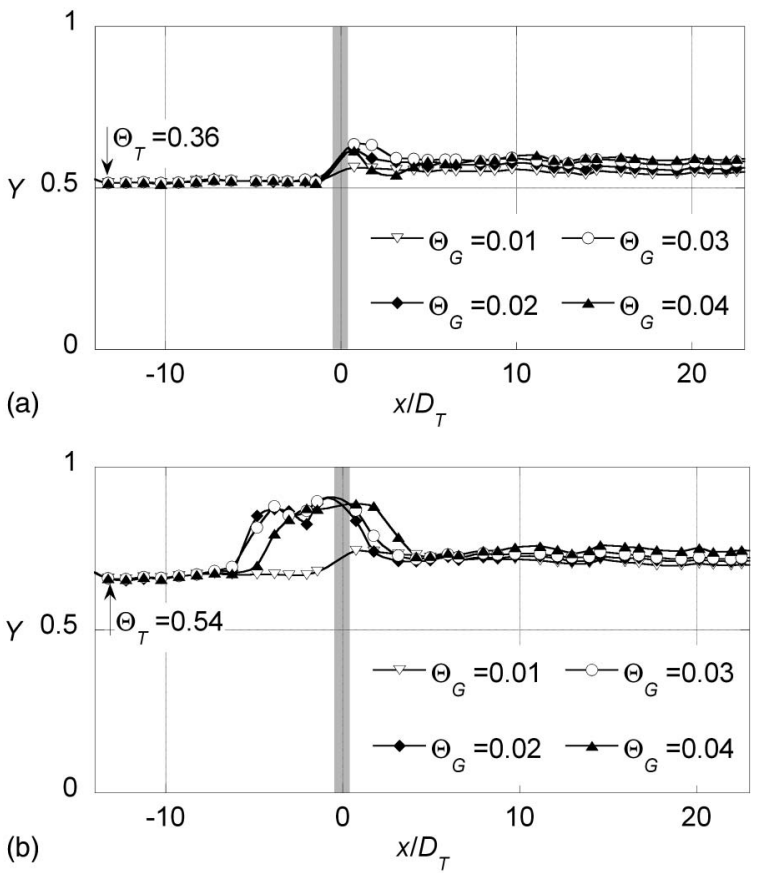

Fig. 5. Tunnel flow surface profiles $Y\left(x / D_{T}\right)$ at junction vicinity for final design: (a) tunnel design discharge condition; (b) tunnel EF condition

is small to minimize the thickness of the water "sheet" supplied into the tunnel. This sheet attaches to the smooth tunnel surface as annular flow, analogous to vortex drop shaft flow. The inflow impacts the tunnel flow laterally, whereas the resulting shear layer mixes the two flows in the streamwise direction, such that shock waves remain only further downstream. No tunnel choking was observed for all discharge combinations tested up to EF (Fig. 5). Upstream of the junction, a short tunnel reach of length $6 D_{T}$ was affected by undular flow under the EF condition of both branches, whereas hardly any effect was visible for the design discharge condition. Fig. 6 indicates small air entrainment from the drop shaft into the tunnel flow. For small $Q_{G}$, the flow is not attached to the tunnel surface but falls as a free jet onto the tunnel flow, without

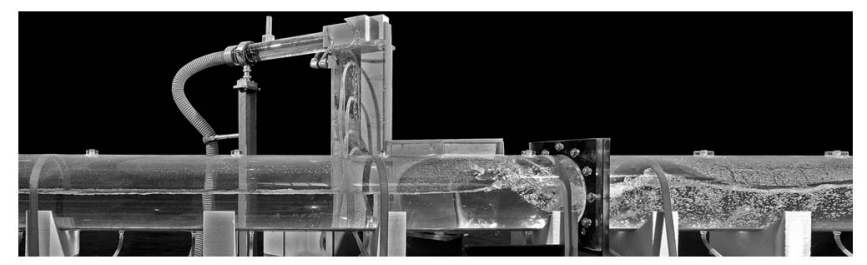

(a)

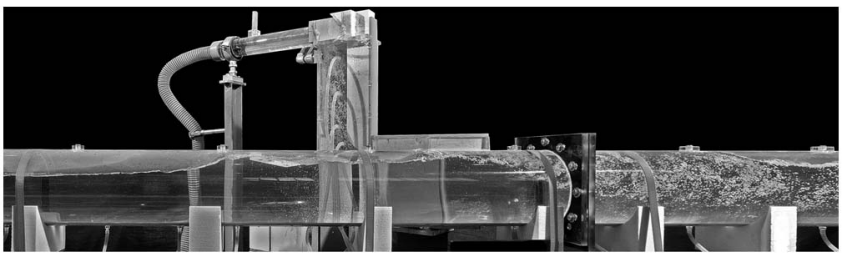

(b)

Fig. 6. Tunnel flow pattern under: (a) design discharge condition; (b) EF condition for final design; the tunnel flow is affected upstream of the junction yet without choking 
tunnel flow choking. For $Q_{G}=0$, the tunnel flow remains unaffected for all $Q_{T}$ because of the high injector position.

The injector cross section also regulates the elevation of the free water elevation in the drop shaft. A maximum injector cross section results from the required minimum value of $V_{G}$ and a minimum cross section by the shaft height, as the supply conduit should not be submerged. The optimized injector generates in the shaft a free surface located $2.8 w$ above the tunnel ceiling for design discharge and 7.2w for EF condition, respectively. As a consequence,

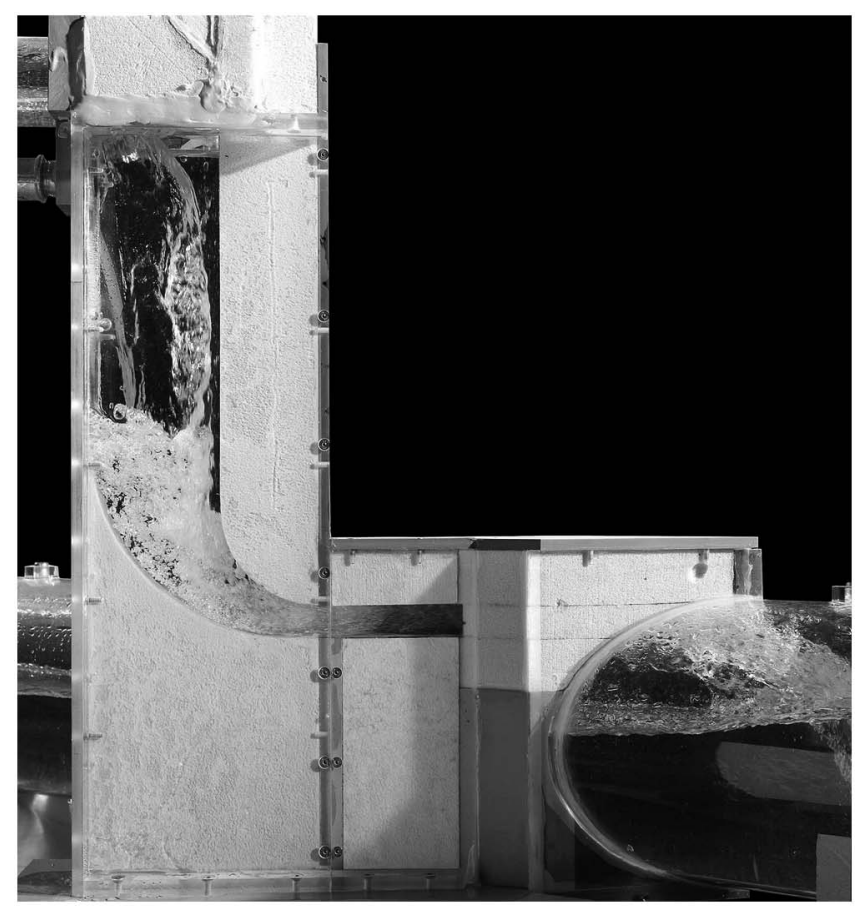

(a)

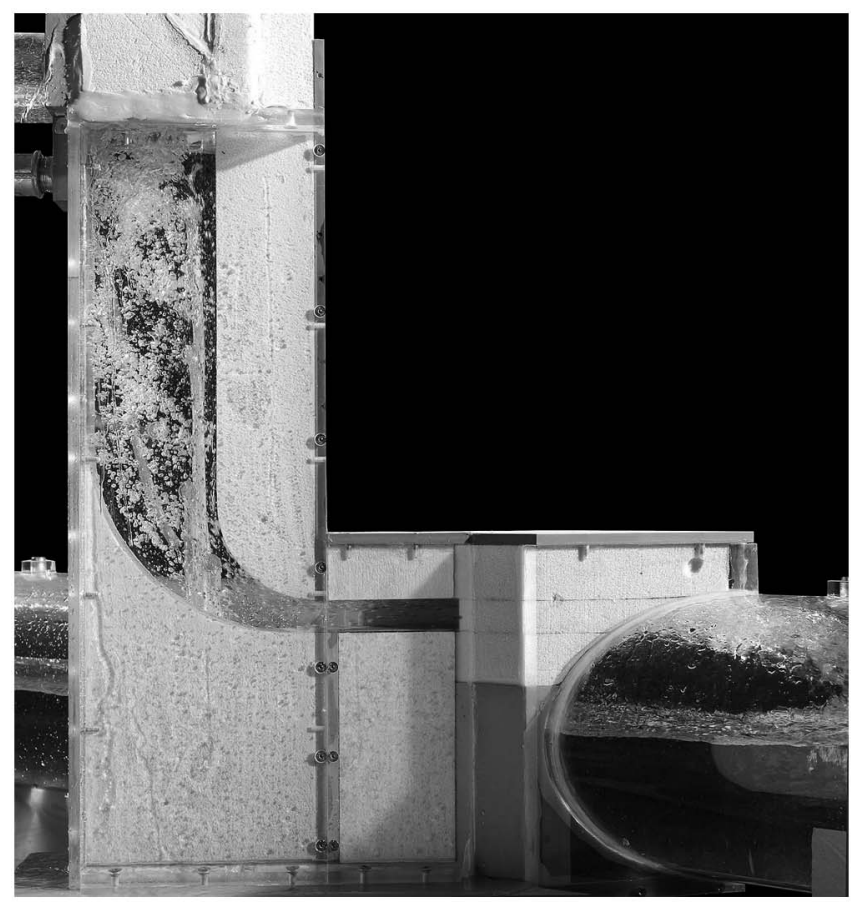

(b)

Fig. 7. Flow pattern in drop shaft with $Q_{T}, 100$ for $Q_{G}=$ : (a) design discharge condition with attached flow and air cavity; (b) EF condition a freeboard of $0.25 w$, equal to $0.25 \mathrm{~m}$, remains to the conduit bottom. Although this is a small value, a free end overfall was observed there for discharges up to EF without any backwater effect in the supply conduit.

In the drop shaft, a 0.7 to $0.8 w$ thick jet attached to the opposite wall, and an air cavity was generated below the overfall (Fig. 7). This cavity was aerated, as significant subpressures occur otherwise. Along the shear zone between the attached flow and the stagnant water, air is entrained and transported to the injector. To ensure proper shaft aeration, two aeration devices were provided: (1) at the top combined with the manhole cover plate, and (2) below the conduit [Fig. 3(e)]. Air entrained along the shaft was transported into the tunnel, where it detrained to the air zone above the flow surface. A deaeration device in the tunnel downstream of the junction is advisable, to reopen choked tunnel flow for $Q_{T}>$ EF. For this overload flood, such a device would support the transition from pressurized to free surface tunnel flow.

The constructional cost of the proposed final design presumably exceeds that of a standard solution, mainly because of the bend combined with the contraction at the shaft foot and its small dimensions, requiring a steel construction. In addition, a dense thrash rack is provided at the creek inlet to avoid any blockage of the contraction by entrained particles. As the creek inlet consists of a side weir, no abrasion attributable to sand or other particles is expected in the lower shaft and the duct.

An alternative option includes a reduced cross section of the vertical shaft. The zones of the air cavity and the stagnant water are then removed, with a shaft length of only $0.8 w$. This leads to extensive air entrainment along the upper jet trajectory, combined with pulsating shaft flow. The air is transported in pockets rotating around the vertical axis and entrained into the tunnel. At the conduit end overfall, negative pressure heads of some $1.4 w$ were measured. The tunnel flow, however, remained unaffecting by this alternative setup.

\section{Conclusions}

No standard concept exists so far to merge drop shaft flow with free surface tunnel flow at almost critical flow conditions and maximum filling ratios. An obvious approach to this problem includes a vortex drop shaft with a dissipation chamber connected to the tunnel via a free overfall. For maximum discharges, however, this design generates undesirable flow choking of both the tunnel and the chamber. A systematic variation of the cross section connecting the chamber with the tunnel included setups with a slot located at the chamber bottom, the tunnel ceiling, and medium tunnel height, yet without improving the flow conditions.

Model tests indicated that the vortex drop shaft concept had to be replaced for the present case by a drop shaft combined with an injector for acceptable flow conditions in both the drop and the tunnel. The injector generates a thin annular flow sheet attached to the tunnel surface of similar streamwise velocity as the tunnel flow. Despite maximum filling ratios, no tunnel choking was observed. For maximum discharges, an acceptable and short increase of the flow depth upstream and shock waves downstream of the junction were observed. The energy required to accelerate the injector flow was provided by a water cushion in the drop shaft, whose surface elevation is a function of discharge. Because of considerable air entrainment, two aeration devices were provided, beside a deaerating device in the tunnel downstream of the junction.

The optimization process led to the following conclusions:

- An injector generating annular flow results in minimum tunnel flow disturbances, 
- The drop shaft should have a sufficient horizontal cross section generating small flow losses and a free water surface,

- The drop shaft has to be sufficiently aerated to avoid fluctuations and subpressures,

- The injector has to be arranged tangentially at the tunnel ceiling without an offset, and

- The injector velocity $V_{G}$ in the tunnel direction should be slightly higher than the tunnel flow velocity $V_{T}$. For the present case $V_{G} / V_{T} \approx 1.1$.

This case study relates to the Lyssbach diversion tunnel model investigation, in which the principal working conditions such as tunnel dimensions, creek inflow fall head, and discharge spectra were specified. No general parameter variation was conducted, limiting so far the application of these presented results to similar cases with slightly supercritical tunnel flow, $\Theta_{T} \leq 0.54, \Theta_{G} \leq 0.04$, and a relative creek fall head of approximately $1.75 \mathrm{D}$. The injector concept generating annular tunnel flow may presumably also be applied in hydraulic structures with different conditions, whereas the precise geometrical design then has to the derived from individual model tests.

\section{Acknowledgments}

The writers kindly thank Bern Public Works, represented by Mr. Jörg Bucher, for excellent collaboration.

\section{Notation}

The following symbols are used in this paper:

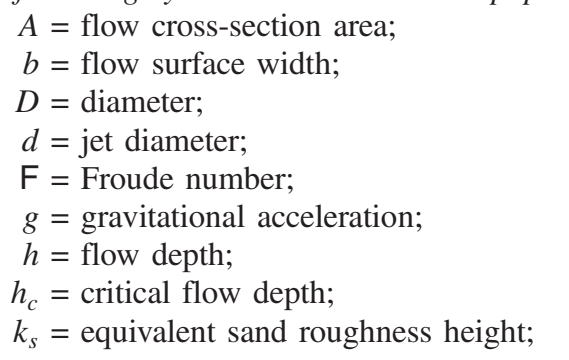

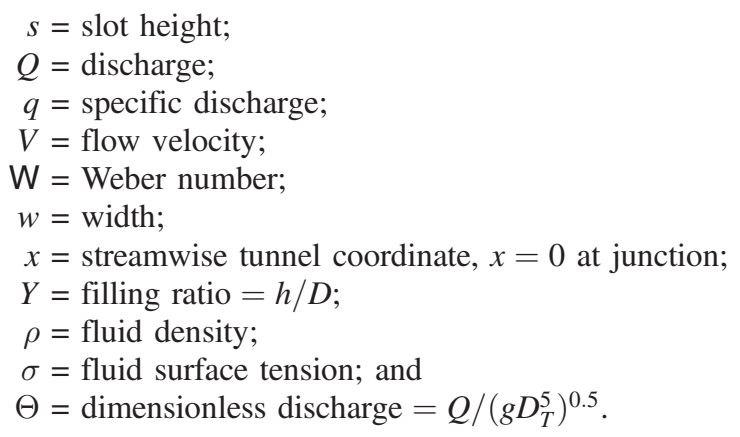

\section{Subscripts:}

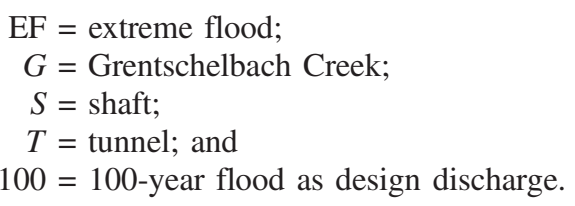

\section{References}

Chanson, H. (2009). "Turbulent air-water flows in hydraulic structures: Dynamic similitude and scale effects." Environ. Fluid Mech., 9(2), $125-142$.

Chow, V. T. (1959). Open-channel hydraulics, McGraw-Hill, New York. Gargano, R., and Hager, W. H. (2002). "Undular hydraulic jumps in circular conduits." J. Hydraul. Eng., 128(11), 1008-1013.

Hager, W. H. (1990). "Vortex drop inlet for supercritical approach flow." J. Hydraul. Eng., 116(8), 1048-1054.

Hager, W. H. (1999). Wastewater hydraulics, Springer, Berlin.

Sauerbrey, M. (1969). "Abfluss in Entwässerungsleitungen unter besonderer Berücksichtigung der Fliessvorgänge in teilgefüllten Rohren (Conduit flow considering part-full flow characteristics)." Wasser und Abwasser in Forschung und Praxis 1, Erich Schmidt, Bielefeld, Germany (in German).

Volkart, P. (1993). “Absturzbauwerke, Toskammern: Eine Übersicht (Drop shafts and dissipation chambers: An overview)." Verbandsbericht 483, Verband Schweizerischer Abwasserfachleute, Zurich, Switzerland (in German). 